\title{
Vertebroplastie und Kyphoplastie bei osteoporotischen Wirbelkörperfrakturen: Indikationen, Technik, Ergebnisse und Komplikationen
}

\author{
Christian W. Müller, Christian Krettek
}

\section{Zusammenfassung}

Osteoporotische Wirbelkörperfrakturen führen häufig zu anhaltenden Schmerzen und Einschränkungen in der Aktivität und Lebensqualität. Vertebroplastie und Kyphoplastie stellen eine effektive Behandlungsoption dar. Unter Durchleuchtungskontrolle erfolgt minimalinvasiv eine Zementaugmentation des Wirbelkörpers, die eine innere Stabilisierung des Wirbelkörpers und damit eine Schmerzreduktion bewirkt. Bei der Kyphoplastie erfolgt zusätzlich eine Aufrichtung des Wirbelkörpers mittels Ballonkatheter. Für die Kyphoplastie wurde in kontrollierten randomisierten Studien eine signifikante Schmerzreduktion und Verbesserung der Lebensqualität nachgewiesen. Zementaustritte aus dem Wirbelkörper können jedoch zu seltenen, aber schwerwiegenden Komplikationen wie Lungenembolien und Nervenschäden führen.

\section{Einleitung}

Die Osteoporose stellt in Europa und den USA mittlerweile eine der bedeutendsten Volkskrankheiten dar, allein in Deutschland leiden bis zu 7,8 Millionen Menschen über 50 Jahre daran [1]. Häufig wird die Osteoporose erst durch eine Fraktur offenbar. Wirbelkörperkompressionsfrakturen stellen mit etwa der Hälfte die häufigsten der osteoporotischen Frakturen dar. Mit 1,4 Millionen osteoporotischen Wirbelkörperfrakturen jährlich wird allein bei den 50-79-Jährigen in Europa gerechnet [2]. Bis vor wenigen Jahren sind diese Frakturen in aller Regel konservativ, d.h. mittels Schmerzthera-

OP-JOURNAL 2009; 25: 28-32

(c) Georg Thieme Verlag KG Stuttgart · New York DOI 10.1055/s-0029-1185556

Vertebroplasty and Kyphoplasty for Osteoporotic Vertebral Compression Fractures: Indications, Technique, Results and Complications

Osteoporotic vertebral compression fractures frequently lead to persisting pain and impair daily activities and quality of life. Vertebroplasty and kyphoplasty are efficient treatment options. Under fluoroscopic guidance the vertebrae are filled with bone cement in a minimally invasive manner. This internal casting leads to a reduction of pain. Kyphoplasty additionally aims to correct the kyphotic deformation of the broken vertebra via the introduction and inflation of a balloon catheter. Randomised controlled trials have shown signficant improvements in pain and quality of life for patients after undergoing kyphoplasty. Cement leakage may lead to rare but severe complications such as pulmonary embolism and nerve palsies.
Dieses kann gesundheitliche Einschränkungen auf anderen Gebieten nach sich ziehen. So begünstigt die Immobilisierung die Entwicklung von Pneumonien, Thrombosen, Embolien, Muskelschwund, Obstipation und Dekubiti. Bei den meist älteren Patienten kann so eine verhängnisvolle „Abwärtsspirale“ entstehen. Um diese Entwicklung rechtzeitig zu durchbrechen, ist daher in erster Linie eine suffiziente Schmerztherapie erforderlich, die häufig medikamentös allein nicht in befriedigendem Ausmaß gelingt.

1987 publizierten Galibert und Deramond die Vertebroplastie als neues Verfahren zur Behandlung schmerzhafter Wirbelkörperhämangiome [4]. Später wurde die Operationsmethode auf die Behandlung osteoporotischer Wirbelkörperfrakturen ausgeweitet. Dies stellt heute die häufigste Indikation dar. Das Prinzip der Vertebroplastie besteht darin, durch Zementinjektion in den gebrochenen Wirbelkörper eine innere Stabilisierung herbeizuführen. Dadurch werden die Mikrobewegungen im Wirbelkörper aufgehoben, welche ansonsten zu einer permanenten Reizung des an Schmerzfasern reichen Periosts führen.

pie und Krankengymnastik, mitunter auch mit Bettruhe oder mit Korsett behandelt worden. Während ein Teil der Patienten mit osteoporotischen Wirbelkörperfrakturen unter konservativer Therapie innerhalb einiger Wochen eine deutliche Beschwerdebesserung erfahren, leiden andere dieser Patienten mitunter viele Monate an stärksten Rückenschmerzen, die zu erheblichen Einschränkungen der Mobilität und Lebensqualität führen. In einer aktuellen schwedischen Studie klagten 12 Monate nach dem Wirbelkörperbruch noch 76\% der Patienten über „erhebliche“ Rückenschmerzen [3].

Osteoporotische Wirbelkörperfrakturen führen häufig zu anhaltenden Schmerzen und Minderung der Lebensqualität.
1998 wurde mit der Kyphoplastie eine Modifikation der Vertebroplastie vorgestellt, bei der ein Ballonkatheter in bracht wird und damit der meist keilförmig deformierte Wirbelkörper zunächst (teilweise) wiederaufgerichtet wird, bevor dann Knochenzement in die durch den Ballonkatheter erzeugte Höhle im Wirbelkörper eingebracht wird. Die Kyphoplastie soll so über die Schmerzlinderung hinaus eine Wiederherstellung der Wirbelkörperform und damit des Wirbelsäulenprofils bewirken. So sollen zusätzlich negative Folgen durch eine zunehmende Rundrückenbildung verhindert werden. den gebrochenen Wirbelkörper einge- 


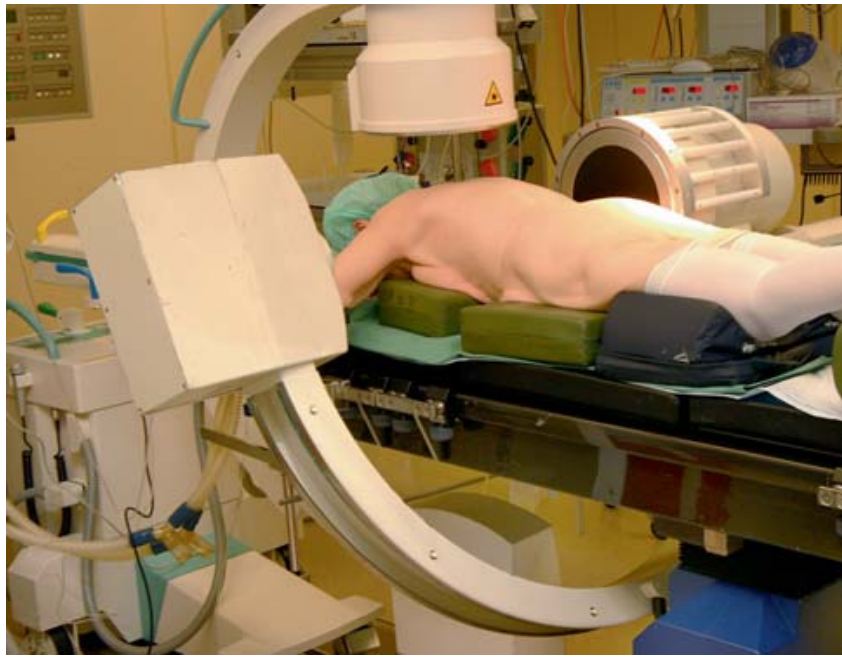

Abb. 1 OP-Setup Kyphoplastie: Bauchlagerung, Arme nach kopfwärts gelagert, Bildwandler für den seitlichen Strahlengang fest, Bildwandler für den a.-p.Strahlengang beweglich von kopfwärts (nur möglich, wenn Fraktur nicht zu weit kaudal gelegen und Patient nicht zu groß).

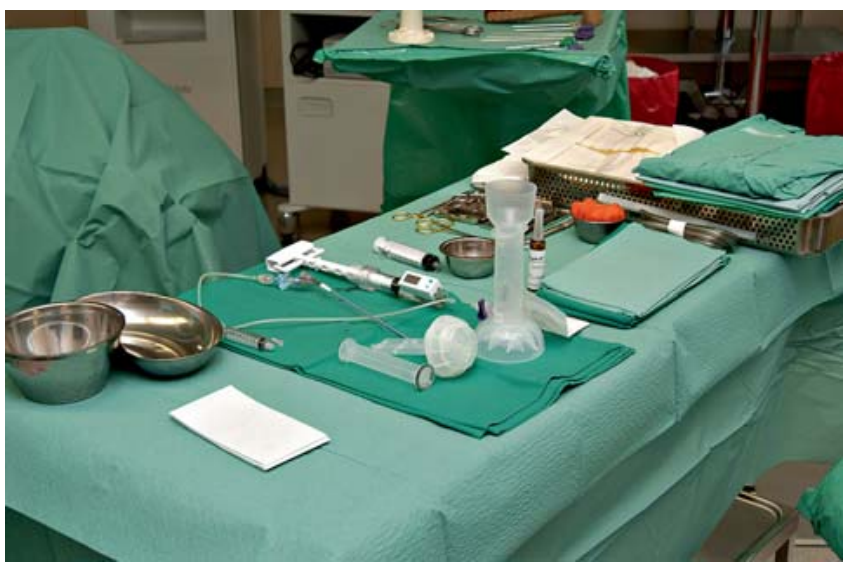

Abb. 2 Instrumentarium Kyphoplastie.

\section{Indikationen}

Als wichtigste Indikationen für eine Zementaugmentation eines Wirbelkörpers, also eine Vertebro- oder Kyphoplastie, werden angesehen:

- schmerzhafte osteoporotische Sinterungsfrakturen an BWS und LWS (häufigste Indikation)

- schmerzhafte Wirbelkörpermetastasen mit oder ohne Fraktur bei multipel metastasiertem Grundleiden

- schmerzhafte Osteolysen des Wirbelkörpers bei Plasmozytom (multiples Myelom)

- schmerzhafte oder stabilitätsgefährdende Wirbelkörperhämangiome

Bei schmerzhaften traumatischen Wirbelkörperkompressionsfrakturen wird fallweise die Indikation zur Kyphoplastie auch ohne begleitende Osteoporose gestellt, allerdings muss hier bei den in der Regel jüngeren Patienten besonders sorgfältig gegenüber der Notwendigkeit einer dorsalen und/oder ventralen Spondylodese (Versteifungsoperation) einer- seits oder der konservativen Behandlung andererseits abgewogen werden.

Eine relative Kontraindikation stellt eine Läsion der Hinterkante des betroffenen Wirbelkörpers dar, weil hier das Risiko eines Zementaustritts in den Spinalkanal erheblich erhöht ist. Kontraindikationen sind weiterhin:

- nicht schmerzhafte Wirbelkörperdeformitäten

- fehlende Übereinstimmung von klinischen und radiologischen Befunden, insbesondere bei erheblichen degenerativen Schäden

- Wirbelkörperdeformitäten, die technisch eine Durchführung nicht erlauben (z.B. Vertebra plana)

- mit kurativer Zielsetzung operable Tumoren und Metastasen

- lokale oder systemische Infektionen

- unbehandelte Gerinnungsstörungen

Osteoporotische Wirbelkörperfrakturen sind die häufigste Indikation für Vertebro- und Kyphoplastie. Präoperativ muss eine Übereinstimmung der klinischen und radiologischen Befunde bestehen.

\section{Technik}

Während vor allem die Vertebroplastie teilweise in Analgosedierung und Lokalanästhesie durchgeführt wird, ist insbesondere bei der Kyphoplastie die Intubationsnarkose eindeutig vorzuziehen. Dies gewährleistet zum einen eine korrekte und gleichbleibende Lagerung während der Operation, zum anderen ermöglicht die Muskelrelaxation eine bessere lagerungsbedingte Wiederaufrichtung des gebrochenen Wirbelkörpers. Schließlich sind sowohl die notwendige Umlagerung auf den Operationstisch als auch die Reposition, sei es durch Lagerung oder durch Entfaltung des Ballonkatheters, ausgesprochen schmerzhaft, sodass auch in dieser Hinsicht die Intubationsnarkose die deutlich patientenfreundlichere Methode darstellt.

Die Operation wird in Bauchlage durchgeführt. Eine Lagerung im leichten Durchhang, also in Hyperlordose, führt bei frischen Wirbelkörperfrakturen bereits zu einer teilweisen Wiederaufrichtung des gebrochenen Wirbelkörpers. Die Unterarme werden auf Armstützen kopfwärts gelagert. Dabei sollten die Armstützen möglichst weit kopfwärts am Operationstisch angebracht werden, um die Handhabung des Bildwandlers zu erleichtern. Es wird nun zunächst der betroffene Wirbelkörper im antero-posterioren und im seitlichen Strahlengang dargestellt. Die Lagerung wird so optimiert, dass der Wirbelkörper exakt in den beiden Ebenen im Bildwandler dargestellt ist und insbesondere die Pedikel des Wirbelkörpers gut erkennbar sind. Wir bevorzugen die Verwendung von 2 Bildwandlern, die in die Abdeckung integriert werden, sodass zu jedem Zeitpunkt die Durchleuchtungskontrolle in 2 Ebenen erfolgen kann. Alternativ kann jedoch auch mit einem Bildwandler gearbeitet werden, mit dem während der Operation dann allerdings häufig „durchgeschwenkt“" werden muss. Abb. 1 zeigt die Lagerung auf dem Bauch und die Position der 2 Bildwandler. Abb. 2 zeigt den Instrumententisch, Abb. 3 das GesamtSetup im OP.

Intubationsnarkose und Verwendung von 2 Bildwandlern erleichtern die Durchführung der Operation.

Es empfiehlt sich, die Lokalisation des betroffenen Wirbelkörpers auf der Haut zu markieren, bevor das Operationsfeld abgewaschen und abgedeckt wird. Der Hautschnitt erfolgt durch Stichinzision 
knapp lateral und kranial der Begrenzung des Pedikels. Mit dem Klemmchen wird stumpf auf den Pedikel zugegangen. Meist erfolgt der Zugang transpedikulär, lediglich im Bereich der mittleren und oberen BWS ist der extrapedikuläre Zugang vorteilhafter. Es wird dann zunächst eine Yamshidi-Nadel unter fluoroskopischer Kontrolle in 2 Ebenen bis über die Hinterkante hinaus in den Wirbelkörper vorgetrieben, wobei sorgfältig darauf geachtet werden muss, die Pedikelwand nicht zu perforieren. Anschließend wird über die Yamshidi-Nadel ein stumpfer Spickdraht eingebracht und bis in das ventrale Drittel des Wirbelkörpers mit vorsichtigen Hammerschlägen eingebracht. Über den Spickdraht wird dann eine Arbeitskanüle bis in die Wirbelkörperhinterwand vorgetrieben. Bei der Vertebroplastie erfolgt nun über diese Arbeitskanüle die Applikation des Knochenzements, in der Regel Polymethylmethacrylat (PMMA). Bei der Kyphoplastie wird über die Arbeitskanüle mit einem Bohrer ein dünner Hohlraum in den Wirbelkörper geschnitten, über den anschließend der Ballonkatheter eingebracht wird. Röntgendichte Markierungen ermöglichen die Lagekontrolle in der Durchleuchtung. Üblicherweise erfolgt dieses Vorgehen über beide Pedikel des Wirbelkörpers. Anschließend werden nun die Ballonkatheter unter Druck mit einem Kontrastmittel gefüllt. Die Ausbreitung der Ballone erfolgt unter Durchleuchtungskontrolle so lange, bis die Ballone die innere Begrenzung des Wirbelkörpers erreichen. Abhängig vom Frakturalter kommt es dabei meist zu einer (teilweisen) Wiederaufrichtung des Wirbelkörpers. Druck und erreichtes Volumen im Ballon sind dabei jederzeit ablesbar. Nach Entfernen der Ballonkatheter wird über die Arbeitskanülen ein hochvisköser Knochenzement in der zuvor durch die Ballonkatheter bestimmten Menge appliziert. Es ist entscheidend, das Erreichen einer sehr zähflüssigen Konsistenz abzuwarten, um das Risiko für Zementaustritte aus dem Wirbelkörper gering zu halten. Die Applikation des Zements muss unter engmaschiger Durchleuchtungskontrolle erfolgen, um im Fall eines Zementaustritts die Verabreichung sofort stoppen zu können. Wenn das zuvor bestimmte Volumen verabreicht ist, wird der Beginn des Aushärtens abgewartet, in der Regel wenige Minuten, dann werden die Arbeitskanülen entfernt, die Zugangswunden gespült und mit Rückstich-Hautnähten versorgt.

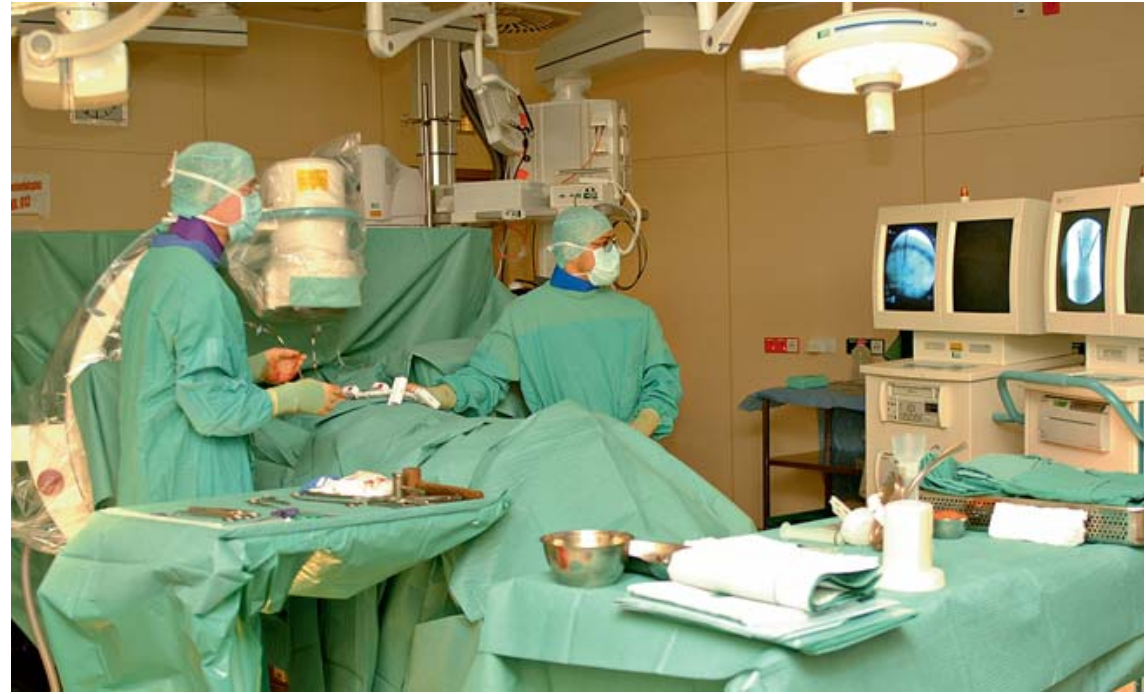

Abb. 3 OP-Setup nach Abdeckung: Bildwandler für den seitlichen Strahlengang in der Abdeckung integriert, Bildwandler für den a.-p.-Strahlengang beweglich von schräg-kopfwärts.

Die Patienten können postoperativ am gleichen Tag mobilisiert werden, einige Autoren empfehlen die Bettruhe für einige Stunden, um die Entwicklung eines Wundhämatoms zu reduzieren. Ein Großteil der Patienten berichtet bereits am ersten postoperativen Tag über eine deutliche Beschwerdebesserung.

\section{Ergebnisse}

$\mathrm{Zu}$ den klinischen Ergebnissen von Vertebroplastie und Kyphoplastie liegen weit über 200 publizierte klinische Studien vor, die jedoch zum allergrößten Teil keine Kontrollgruppe beinhalten und somit nicht den Kriterien einer evidenzbasierten Medizin entsprechen. Nahezu alle Autoren beschreiben darin eine erhebliche Besserung der Beschwerden nach der Operation, wobei sowohl für die Vertebroplastie als auch für die Kyphoplastie regelmäßig „Erfolgsraten“ von über $90 \%$ angegeben werden.

In 2 aktuellen Metaanalysen zur Kyphoplastie in der Behandlung von Wirbelkörperkompressionsfrakturen wurden 8 vergleichende Studien zur Kyphoplastie (3 gegen konservative Therapie und 5 gegen Vertebroplastie) gefunden. Die Autoren fassen als Ergebnis zusammen, dass die Kyphoplastie der konservativen Therapie in Bezug auf die Schmerzreduktion und die Lebensqualität der konservativen Behandlung überlegen und der Vertebroplastie mindestens ebenbürtig sei. Ein signifikanter Unterschied bestand in Bezug auf Zementleckagen, die bei der Vertebroplastie häufiger auftraten. Zusammenfassend wurde eine Klasse-III-
Evidenz für die Effektivität von Kyphoplastie und Vertebroplastie in der Behandlung von symptomatischen Wirbelkörperkompressionsfrakturen nach fehlgeschlagener konservativer Therapie gesehen. Bei insgesamt günstigem Verhältnis von Operationsergebnis und Risiken wurde der Kyphoplastie ein besseres Risikoprofil bescheinigt $[5,6]$.

Die Kyphoplastie führt bei Patienten mit osteoporotischen Wirbelkörperfrakturen zu einer signifikanten Verbesserung der Schmerzen und Lebensqualität gegenüber konservativer Therapie allein.

In der internationalen, prospektiven, randomisierten Multicenterstudie zum Vergleich der Kyphoplastie und der nicht operativen Behandlung bei akut schmerzhaften Wirbelkörperfrakturen, deren Ergebnisse bislang nur in Abstraktform publiziert sind, wurden durchweg Vorteile in der Kyphoplastiegruppe gefunden: Auf der visuellen Analogschmerzskala von 0 bis 10 gaben Patienten nach Kyphoplastie nach 7 Tagen einen um 2,2 Punkte niedrigeren Schmerzwert an als Patienten nach konservativer Therapie. Auch nach 12 Monaten bestand noch ein Vorteil von 0,9 Punkten. Auch die verschiedenen Tests zur Lebensqualität (Short Form 36, Roland Morris Disability Questionnaire) ergaben zu allen Untersuchungszeitpunkten bis 12 Monate signifikant bessere Ergebnisse für die Patienten nach Kyphoplastie im Vergleich zu den konservativ behandelten Patienten [7]. In nicht randomisierten Studien wurde noch bis zu 36 Monate nach Kyphoplastie eine signifikante Verbes- 

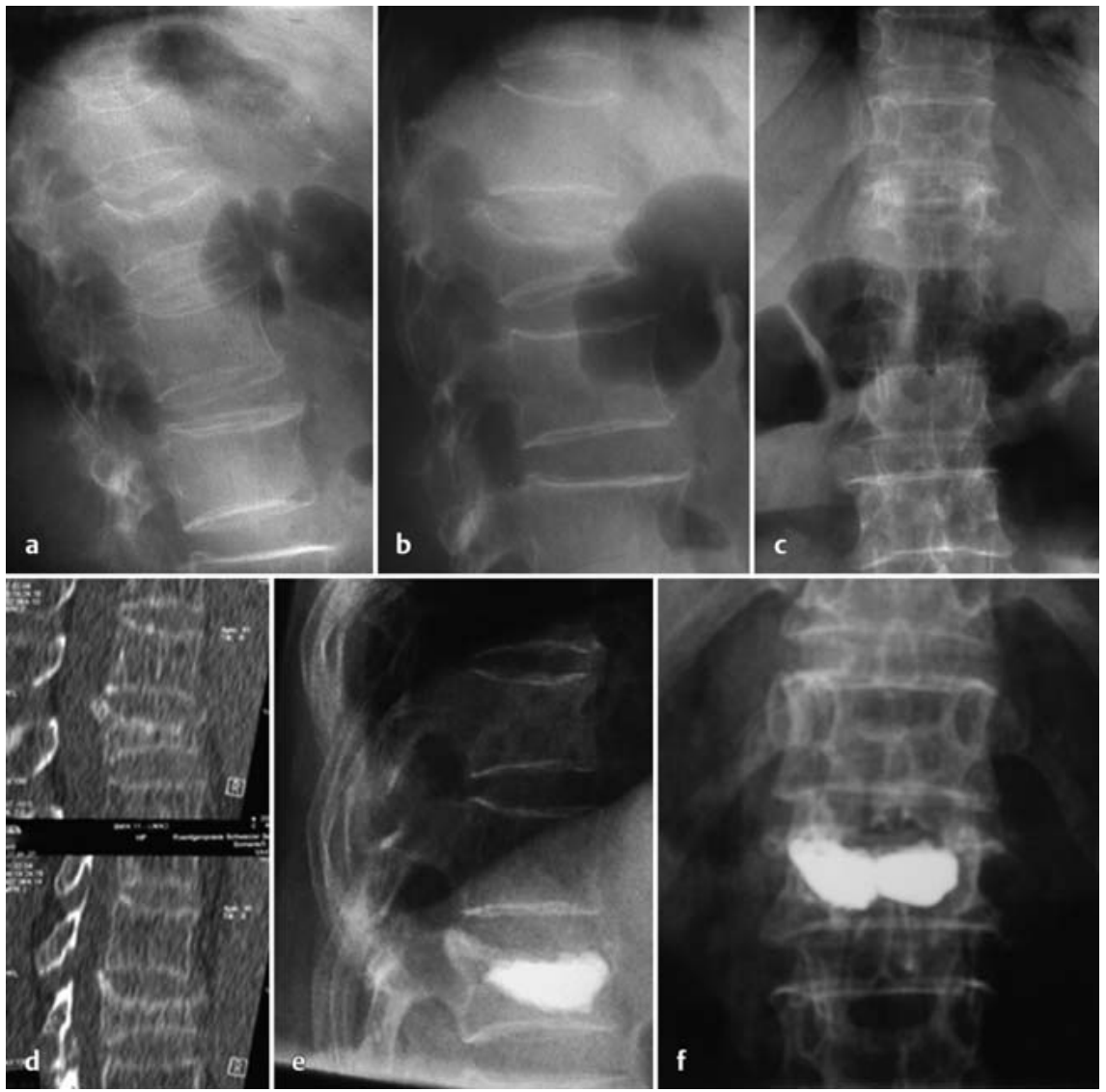

Abb. 4 a bis f Osteoporotische LWK-1-Kompressionsfraktur, Kyphoplastie LWK 1 (s. Text).

serung im Vergleich zur Kontrollgruppe beschrieben [8].

Zur Evaluierung der Vertebroplastie sind gegenwärtig 2 randomisierte Studien in Planung bzw. Durchführung, Ergebnisse stehen noch aus $[9,10]$.

\section{Fallvorstellung}

Abb. 4 zeigt die Röntgenbilder einer 77jährigen Patientin aus dem eigenen Krankengut. Sie stellte sich mit akuten Rückenschmerzen ohne erinnerliches Trauma in der Notaufnahme vor. Die initiale Röntgendiagnostik (a) zeigte eine Kompressionsfraktur des 1. Lendenwirbelkörpers. Nach zunächst konservativer Behandlung zeigt die Verlaufskontrolle nach 10 Tagen eine zunehmende Sinterung $(\mathbf{b}, \mathbf{c})$. Die Rückenschmerzen wurden weiterhin mit einem subjektiven Schmerzwert von 9 von 10 angegeben. Die Computertomografie bestätigte die Kompressionsfraktur des 1. Lendenwirbelkörpers (d). Es wurde die Indikation zur Kyphoplastie des 1. LWK gestellt. Die postoperativen Röntgenbilder zeigen eine teilweise Wiederaufrichtung des Wirbelkörpers und eine regelrechte Ze- mentauffüllung ohne Zementaustritt (e, f). Die Schmerzen am 1. postoperativen Tag wurden mit 1-2 von 10 angegeben. Die Patientin konnte am 3. postoperativen Tag aus der stationären Behandlung entlassen werden.

\section{Komplikationen}

Die wichtigste Komplikation stellt der intraoperative Zementaustritt aus dem zementaugmentierten Wirbelkörper dar. In Studien werden bei 5 bis $20 \%$ der Kyphoplastien und bei 50 bis $90 \%$ der Vertebroplastien Zementaustritte beschrieben [6]. Die erheblich höhere Rate bei der Vertebroplastie ist zum einen auf die niedrigere Viskosität und zum anderen auf den notwendigen höheren Druck bei Verabreichung des Zements zurückzuführen. Die allermeisten Zementaustritte bleiben ohne klinische Folgen, abhängig vom genauen Ort und der Menge des Zementaustritts sind allerdings schwere Komplikationen möglich: Ein Abschwemmen von Zement über die Segmentgefäße kann zu einer fulminanten Lungenembolie führen, tödliche Verläufe sind in der Literatur beschrieben [11]. Lungenembolien können auch als
Fettembolien auftreten. Zementaustritte im Bereich der Spinalnerven und des Rückenmarks können zu Nervenreizungssyndromen, Lähmungen und, als Einzelfälle beschrieben, kompletter Querschnittslähmung führen [12]. Zementaustritte in benachbarte Bandscheiben führen in der Regel nicht unmittelbar zu Symptomen, es wird allerdings angenommen, dass dies einer Degeneration der Bandscheibe zumindest Vorschub leistet.

OP-Komplikationen sind selten, aber mitunter sehr schwerwiegend.

Weitere, seltenere Komplikationen sind Blutungen, Verletzungen der neuronalen Strukturen durch den Zugang und Infektionen. Für die Kyphoplastie wurde in der Metaanalyse von Taylor eine Rate an Lungenembolien mit 1,7 von 1000 berechnet, 1,6 von 1000 Patienten erlitten eine Kompression des Rückenmarks und 1,7 eine Reizung der peripheren Nerven. 1,3 von 1000 Patienten starben innerhalb von 30 Tagen nach der Operation, welches die Autoren im Wesentlichen auf das hohe Alter der Patienten und den hohen Anteil an Krebspatienten zurückführten [5]. Vergleichbare, systematische Zahlen für die Vertebroplastie liegen nicht vor, allerdings muss anhand der vorliegenden Veröffentlichungen hier von einer insgesamt höheren Komplikationsrate ausgegangen werden.

In der Vergangenheit wurde vielfach angenommen und auch mit Studien belegt, dass es in der Folge der Zementaugmentierung gehäuft zu sog. Anschlussfrakturen, d.h. Frakturen in benachbarten Wirbelkörpern komme. Größere Studien haben dies allerdings nicht bestätigen können, insgesamt wurde in den beschriebenen Metaanalysen sogar eine Verringerung der Gesamtrate an neu aufgetretenen Wirbelkörperfrakturen im Nachbeobachtungszeitraum gefunden $[5,6]$.

\section{Fazit}

Vertebroplastie und Kyphoplastie zeigen überzeugende klinische Ergebnisse in Bezug auf die Schmerzlinderung und Verbesserung der Lebensqualität bei Patienten mit osteoporotischen Wirbelkörperfrakturen. Diese Aussage muss für die Vertebroplastie insoweit eingeschränkt werden, dass die Studienlage im Vergleich zur Kyphoplastie deutlich schwächer ist und prospektive, randomisierte Studien zur Vertebroplastie bislang nicht 
vorliegen. Die Kyphoplastie bietet prinzipiell den Vorteil einer teilweisen Wiederherstellung der Wirbelkörperform, ein diesbezüglicher klinischer Zusatznutzen wird angenommen, ist aber letztlich nicht bewiesen. Der Nachteil der Kyphoplastie liegt in den erheblich höheren Materialkosten.

Insgesamt sind Komplikationen, v.a. bei der Kyphoplastie, selten, die Auswirkungen können allerdings beträchtlich sein. Nicht zuletzt deswegen scheint es geboten, dass die Anwendung der Zementaugmentierung in Kliniken erfolgt, in denen zum einen eine ausreichende $\mathrm{Er}$ fahrung mit den Verfahren vorliegt und zum anderen auch die möglichen Komplikationen effektiv behandelt werden können.

Abschließend bleibt festzustellen, dass Vertebroplastie und Kyphoplastie bei osteoporotischen Wirbelkörperfrakturen immer durch eine medikamentöse Osteoporosetherapie, in der Regel Calcium, Vitamin D und ein Biphosphonat, ergänzt werden sollten, um das Risiko für weitere osteoporotische Frakturen zu vermindern.

\section{Literatur}

${ }^{1}$ Anonymous. Incidence of vertebral fracture in Europe: results from the European Prospective Osteoporosis Study (EPOS). J Bone Miner Res 2002; 17: 716-724

2 Haussler B, Gothe H, Gol D et al. Epidemiology, treatment and costs of osteoporosis in Germany - the BoneEVA Study. Osteoporos Int 2007; 18: 77-84

3 Suzuki N, Ogikubo O, Hansson T. The course of the acute vertebral body fragility fracture: its effect on pain, disability and quality of life during 12 months. Eur Spine J 2008; 17: 1380-1390

${ }^{4}$ Galibert P, Deramond H, Rosat P et al. [Preliminary note on the treatment of vertebral angioma by percutaneous acrylic vertebroplasty]. Neuro-Chirurgie 1987; 33: 166-168

5 Taylor RS, Fritzell P, Taylor RJ. Balloon kyphoplasty in the management of vertebral compression fractures: an updated systematic review and meta-analysis. Eur Spine J 2007; 16: 1085-1100

${ }^{6}$ Taylor RS, Taylor RJ, Fritzell P. Balloon kyphoplasty and vertebroplasty for vertebral compression fractures: a comparative systematic review of efficacy and safety. Spine 2006; 31 : 2747-2755

7 Müller CW, Wardlaw D, Bastian L et al. An international multicenter randomized comparison of balloon kyphoplasty and nonsurgical management for patients with acute vertebral compression fractures (FREE study): one year results [Abstrakt 3. Deutscher Wirbelsäulenkongress]. Eur Spine J 2008; 17: 1540-1633

${ }^{8}$ Kasperk C, Hillmeier J, Noldge G et al. Treatment of painful vertebral fractures by kypho- plasty in patients with primary osteoporosis: a prospective nonrandomized controlled study. J Bone Miner Res 2005; 20: 604-612

${ }^{9}$ Klazen C, Verhaar H, Lampmann L et al. VERTOS II: Percutaneous vertebroplasty versus conservative therapy in patients with painful osteoporotic vertebral compression fractures; rationale, objectives and design of a multicenter randomized controlled trial. Trials 2007; 8: 33

${ }_{10}$ Buchbinder R, Osborne RH, Ebeling PR et al. Efficacy and safety of vertebroplasty for treatment of painful osteoporotic vertebral fractures: a randomised controlled trial [ACTRN012605000079640]. BMC Musculoskelet Disord 2008; 9: 156

${ }^{11}$ Chen HL, Wong CS, Ho ST et al. A lethal pulmonary embolism during percutaneous vertebroplasty. Anesthesia and analgesia 2002; 95: 1060-1062

12 Lee BJ, Lee SR, Yoo TY. Paraplegia as a complication of percutaneous vertebroplasty with polymethylmethacrylate: a case report. Spine 2002; 27: E419-E422

Dr. med. Christian W. Müller Facharzt für Chirurgie Prof. Dr. med. Christian Krettek, FRACS

Ärztlicher Direktor

Unfallchirurgische Klinik

Medizinische Hochschule Hannover

Carl-Neuberg-Str. 1

30625 Hannover

mueller.christian@mh-hannover.de 